\title{
Overview of Method for Reducing Grounding Resistance of Substation Grounding System
}

\author{
Bin Tang ${ }^{1}$, Xu Sun ${ }^{2}$, Jiayong Zou ${ }^{3, \mathrm{a}}$ \\ ${ }^{1}$ Fujian Provincial Electric Power Company Limited, State Grid \\ Corporation of China, Fuzhou, China \\ ${ }^{2}$ Guangzhou Electric Power Design Institute, Guangzhou, China \\ ${ }^{3}$ Southwest Electric Power Design Institute, Chengdu, China \\ azou_jiayong@qq.com
}

\begin{abstract}
.
In order to reduce the grounding resistance of the grounding device, people takes various measures, as shown below: expanding the grounding grid area, exterior grounding, utilizing natural grounding, deep well grounding, local soil replacement and the like. The paper introduces the principles and the application scopes of these methods.
\end{abstract}

Keywords: exterior grounding, deep well grounding, substation, grounding resistance and substation.

\section{Introduction}

There are the following two main factors that influence the grounding resistance of the grounding grid: soil resistivity and grounding grid size; if the soil resistivity is low, the grounding resistance is low; the grounding grid size includes the transverse floor area of the grounding grid and the longitudinal span depth of the grounding grid. Thereby, there are three ideas of reducing the grounding resistance of the grounding grid, as shown below: changing the soil resistivity, expanding the transverse floor area of the grounding grid and increasing the longitudinal span depth of the grounding grid. The current resistance reduction problems of the grounding grid can be solved by the above three ideas [1-19].

\section{Method for Reducing the Resistance through Changing the Soil Resistivity}

Resistance Reducing Agent. With the adoption of the resistance reducing agent, the contact resistance between the grounding electrode and the soil is reduced on one hand, and on the other hand, the grounding electrode diameter is increased, and the scattered flow resistance is reduced. In addition, a lot of study has been proved that another principle of the resistance reducing agent for 
reducing the grounding resistance is strong infiltration capacity. As an area in which the resistance reducing agent is infiltrated is formed on the periphery of the grounding electrode, the diffused flow performance of the soil can be improved within a larger scope on the periphery of the grounding device to reduce the diffused flow resistance.

Local Soil Replacement. The soil resistivity directly influences the grounding resistance value. As for some grounding devices located in areas with high soil resistivity, if the resistance is hard to reduce by adopting other methods, the local soil replacement method can be adopted. The soil with lower resistivity or the resistance reducing agent can be used for replacing the high-resistivity soil on the periphery of the grounding devices to obtain lower grounding resistance. This method must be comprehensively compared from the aspect of technical economy before use to avoid economic wastes.

Electrolytic Ion Grounding System and Grounding Module. The electrolytic ion grounding system is characterized in that some ceramic alloy solid compounds are filled into perforated copper pipes or perforated copper alloy pipes, and as electrolytic ion compounds are contained in the pipes, each copper pipe is changed into an electrolytic ion grounding electrode. The grounding module is also added with ions based on coke, so that on one hand, the moisture of the surrounding soil is kept, and on the other hand, the ions release to the periphery, therefore, a role in reducing the soil resistivity can be played.

Seen from the nature of the resistance reducing principles, no obvious difference exits between the electrolytic ion grounding system and the traditional resistance reducing agent in aspect of reducing the grounding resistance of the grounding grid, and only the service life of the resistance reducing agent is prolonged; if the electrolytic grounding electrode array is used for improving the soil resistivity on a large scale, the effect of expanding the diffused flow area to reduce the grounding resistance can be achieved, which is consistent with the resistance reducing effect realized by the local soil replacement method in principle.

\section{Method for Reducing the Resistance through Expanding the Transverse Floor Area of the Grounding Grid}

Exterior grounding. Exterior grounding means a method for connecting the substation main grounding grid with auxiliary grounding grids paved in a certain low-soil resistivity area outside the main grounding grid area to achieve the purpose of reducing the grounding resistance of the whole grounding system. It should be noted that an obvious potential difference exits between the substation and the distant grounding equipment, and particularly, the potential difference is more obvious under high-frequency impact by lightning. It is important to make sure that the main grounding grid is connected with exterior grounding through a plurality of grounding conductors.

Utilizing natural grounding. Natural grounding includes reinforced concrete steel skeletons of surrounding buildings, trash racks at water inlets of hydropower 
stations, gates, water pipes and the like. As these natural grounding electrodes themselves are lower in grounding resistance, before the design of the substation grounding grid, it should be fully considered that these natural grounding electrodes are connected with the main grounding grid to achieve the purpose of reducing the grounding resistance of the grounding grid; particularly, the resistance reducing effect on the hydropower station realized by utilizing natural grounding is fairly obvious, and only less investment is needed. Therefore, not only is the reduction of the grounding resistance by fully utilizing natural grounding easy to realize technically, but also the technical and economic benefits are better.

\section{Method for Reducing the Resistance through Increasing the Longitudinal Span Depth of the Grounding Grid}

Increasing the buried depth of the grounding grid. The buried depth means the distance between the burying position of the horizontal grounding grid and the ground. On the premise that the other parameters of the grounding grid are not changed, although the grounding resistance can be reduced through increasing the buried depth of the grounding grid, the resistance reducing effect is not obvious, in particular to areas with high soil resistivity. Therefore, in general, this method is not adopted in the engineering. The buried depth of the substation grounding grid is about $0.8 \mathrm{~m}$ generally.

Long vertical grounding electrodes, blasting grounding and deep well grounding vertical grounding electrodes, blasting grounding and deep well grounding. If a lower low-resistivity layer exists in the soil in which the grounding system is positioned, long vertical grounding electrodes, blasting grounding and deep well grounding can be adopted.

In general, the soil resistivity is non-uniform along depth and transverse distribution and the resistivity of different depths of soil is different. In an area with high soil resistivity on the surface, the long vertical grounding electrodes are connected with the main grounding grid to effectively reduce the grounding resistance. In an area with an underground aquifer, the grounding electrodes may deeply penetrate through the aquifers, so the resistance reducing effect is better. The diameters of the grounding electrodes can be increased by the grounding well (The grounding rods are added with low-resistivity backfill materials), so the overall grounding resistance is reduced. If the grounding well is connected with the underground aquifer, the seasonal change of the grounding resistance can be reduced, the electrode flow can be increased, and the electrodes cannot be overheated or the backfill materials in the grounding well cannot be dried. Only the lower grounding resistance can be obtained through matching deep well grounding with the low-resistivity materials, and a deep hole blasting cracking-pressure grouting resistance reducing agent method can also be adopted to form a huge low-resistivity area, thereby achieving the resistance reducing purpose. 
Deep inclined shaft grounding. Reducing the grounding resistance only can be considered from two aspects: expanding the area and developing to the horizontal direction; adopting deep vertical grounding electrodes and developing to the depth direction. Deep inclined shaft grounding means that the long vertical electrodes are obliquely embedded into the soil, and the characteristics of diffused flow area expansion and development to the depth direction are combined, which can play a better resistance reducing role when being adopted in the substation grounding engineering of which the grounding grid area is limited.

\section{Application Scopes of Different Resistance Reducing Technologies}

Deep inclined shaft grounding. The application of the resistance reducing agent to the power system grounding engineering becomes less and less. If the resistance reducing agent cannot release ions, and the area where the resistance reducing agent is in is positioned on the periphery of the grounding body, the grounding resistance of the grounding grid cannot be obviously reduced only through reducing the soil resistivity nearby the grounding body in the large grounding grid. In practical engineering, fine earth can be used for replacing the resistance reducing agent to achieve the purpose of reducing the contact resistance between the soil and the grounding body.

Local soil replacement. As for some grounding devices positioned in areas with high soil resistivity, if the resistance is hard to reduce by adopting other methods, the local soil replacement method can also be adopted. The soil with lower resistivity or the low-resistivity materials can be used for replacing the soil with high resistivity on the periphery of the grounding device to obtain lower grounding resistance. However, if the substation needs to be built in a mountainous area or the area where the substation is to be built in is hard to excavate, the method is more difficult to adopt. Therefore, the method must be comprehensively compared from the aspect of technical economy before use to avoid economic wastes.

Electrolytic ion grounding system. In fact, the electrolytic ion grounding system can be used for improving the soil resistivity on the periphery of the grounding system. If a larger area needs to be improved, the soil replacement method had better be adopted. In rocky mountainous areas where the low-resistivity soil is hard to find out or areas which are hard to excavate, the workload caused by the electrolytic ion grounding system is smaller than that caused by the soil replacement method. Therefore, on the premise of ensuring long-term reliability of the electrolytic ion grounding system, the comparison with the local soil replacement method shall be carried out aiming at practical engineering from the aspect of technical economy to avoid economic wastes.

Exterior grounding. Exterior grounding means that a horizontal grounding strip is paved outwards for the substation main grounding grid so as to achieve the purpose of reducing the grounding resistance of the whole grounding system. Exterior grounding can be adopted only under the condition that long-distance horizontal grounding strips are allowed to be paved on the cable trench and the 
substation road, and meanwhile, the step voltage along the exterior horizontal grounding belts must be not more than the safe limit. If the step voltage exceeds the safe limit, an additional insulating layer must be paved to ensure personal safety.

Utilizing natural grounding. Utilizing natural grounding can be adopted only under the condition that an area with lower soil resistivity is found out on the periphery of the main grounding grid. When the substation needs to be built in an area with high soil resistivity, an area with lower soil resistivity may be hard to find out, so grounding reservoirs, wells and the like shall be fully utilized to reduce the grounding resistance. Before the adoption of natural grounding, the step voltage on the periphery must be not more than the safe limit. If the step voltage exceeds the safe limit, an additional insulating layer must be paved to ensure personal safety.

Long vertical grounding electrodes. As for the substation with lower deep soil resistivity than that of the surface soil, the long vertical grounding electrodes are deep into the low-resistivity soil to obviously reduce the grounding resistance. However, as for the substation with higher deep soil resistivity than that of the surface soil, the resistance reducing effect realized by the long vertical grounding electrodes is not obvious, and the resistance reducing effect on the large-area grounding grid is worse. In addition, the vertical grounding electrodes play an obvious role in improving seasonal coefficients, in particular to the effect of frozen soil in winter, so the vertical grounding rod with a length of the 2-3 meters can be densely used.

Deep well grounding. In general, the resistivity of soil closer to the ground is not stable and is changed along with the change of seasonal climate, and the deeper the soil is, the more stale the resistivity is. Particularly, in areas with high soil resistivity and areas in which the grounding device can be not buried by adopting the conventional method, deep well grounding can be connected with the main grounding grid in parallel to effectively reduce the grounding resistance. In places with underground aquifers, in combination with the soil resistivity measurement and the guidance of layered parameters analysis results, the grounding body may deeply penetrate through the aquifer, so that the resistance reducing effect is better. Deep well grounding is not influenced by climate and seasonal conditions, which not only plays a resistance reducing role, but also can overcome the defect that the site is narrow, thereby being an effective method for hydropower station construction in mountainous areas. One of important disadvantages of deep well grounding is that a better resistance reducing role can be played only when the deep well reaches a certain depth.

Blasting grounding. Blasting grounding is particularly suitable for areas where the grounding resistance is hard to reduce by adopting the conventional method, and particularly, in areas with high soil resistivity, the resistance reducing effect realized by the blasting grounding is very obvious. At present, about more than 30 power stations and substation grounding engineering which is difficult in resistance reduction has been solved. The blasting grounding has one of important disadvantages that compared with that of other methods, its expense is higher. 
Deep inclined shaft grounding. Reducing the grounding resistance only can be considered from two aspects: expanding the area and developing to the horizontal direction; adopting deep vertical grounding electrodes and developing to the depth direction. Deep inclined shaft grounding combines the characteristics of diffused flow area expansion and development to the depth direction, which can play a good resistance reducing role when being adopted in the substation grounding project which is limited in grounding grid area.

\section{Conclusion}

The paper systematically introduces the following several common measures for reducing the grounding resistance: expanding the grounding grid area, exterior grounding, utilizing natural grounding, deep well grounding, local soil replacement and the like. The paper not only introduces their principles, but also carefully analyzes their application scopes.

The study in the paper has active significances in improving the scientificity of the substation resistance reduction measures, improving the economy and reducing the cost.

\section{References}

[1] Li Zhongxin, Yuan Jiansheng, Zhang Liping. Numerical analysis of substation grounding systems [J]. Proceedings of the CSEE, 1999, 19(5): $75-79$ (in Chinese).

[2] Zhuang Chi-jie, Zeng Rong, Zhang Bo, et al. Grounding system design method in high soil resistivity regions [J]. High Voltage Engineering. 2008, 34(5):893-897 (in Chinese).

[3] He Jinliang, Kang Peng, Zeng Rong, et al. Grounding system design of Wudaoliang and Tuotuohe substations for $110 \mathrm{kV}$ power transmission engineering of Qinghai-Tibet railway [J]. Power System Technology, 2006, 30(1): 55-59 (in Chinese).

[4] Zeng Rong, Zhuang Chijie, Niu Ben, et al. Measurement of transient electric fields in air gap discharge by integrated electro-optic sensor [J]. IEEE Transactions on Plasma Science. 2013, 41(4): 955-960.

[5] Zhuang Chijie, Zeng Rong. A positivity-preserving scheme for the simulation of streamer discharges in non-attaching and attaching gases [J]. Communications in Computational Physics. 2014, 15(1): 153-178. 
[6] Zhang Wenliang, Zhang Guobing. Discussion and comparison of characteristics of AC-testing supply used for UHVAC [J]. Proceedings of the CSEE, 2007, 27(4): 3-6 (in Chinese).

[7] Zhuang Chijie, Zeng Rong. A local discontinuous Galerkin method for 1.5-dimensional streamer discharge simulations [J]. Applied Mathematics and Computation. 2013, 219(19): 9925-9934.

[8] Zhang Bo, Cui Xiang, Zhao Zhibin, et al. Analysis of grounding grids at large scale substations in frequency domain [J]. Proceedings of the CSEE, 2002, 22(9): 59-63(in Chinese).

[9] Zhuang Chijie, Zeng Rong, Zhang Bo, et al. A WENO scheme for simulating streamer discharge with photoionizations [J]. IEEE Transactions on Magnetics. 2014, 50(2): 7007904.

[10] He Jinliang, Gao Yanqing, Zeng Rong, et al. Optimal design of grounding system considering the influence of seasonal frozen soil layer [J]. IEEE Trans. on Power Delivery, 2005, 20(1): 107-115.

[11] Zhuang Chijie, Zeng Rong, Zhang Bo, et al. 2-D discontinuous Galerkin method for streamer discharge simulations in nitrogen [J]. IEEE Transactions on Magnetics. 2013, 49(5): 1929-1932.

[12] Zeng Rong, Zhuang Chijie, Yu Zhanqing, et al. Challenges and Achievement in Long Air Gap Discharge Research [J]. High Voltage Engineering, 2014, 40(10):2945-2955 (in Chinese).

[13] Zhuang Chijie, Zeng Rong. Research and development on short gap streamer discharge simulation methods [J]. Proceedings of the CSEE. 2012, 32(22): 157-165 (in Chinese).

[14] He Jinliang, Zeng Rong, Gao Yanqing, et al. Seasonal influences on safety of substation grounding system [J]. IEEE Trans. on Power Delivery, 2003, 18(3): 788-795.

[15] Zhuang Chijie, Geng Yinan, Zeng Rong. Discontinuous Galerkin method for short air gap discharge simulations and its applications [J]. High Voltage Engineering. 2013, 39(4): 970-978 (in Chinese).

[16] Chen She, Zhuang Chijie, Zeng Rong, et al. Improved gap factor of large sphere-plane and its application in calculating air gap clearance in UHVDC 
converter station [J]. High Voltage Engineering. 2013, 39(6): 1360-1366 (in Chinese).

[17] Zhuang Chijie, Zeng Rong, Zhang Bo, et al. The optimization of entering route for live working on $750 \mathrm{kV}$ transmission towers by space electric-field analysis [J]. IEEE Transactions on Power Delivery. 2010, 25(2): 987-994.

[18] Zhuang Chijie, Zeng Rong, Zhang Bo, et al. Accelerating the convergence of algebraic multigrid for quadratic finite element method by using grid information and p-multigrid [J]. IEEE Transactions on Magnetics. 2011, 47(5): 1198-1201.

[19] Zeng Rong, Zhuang Chijie, Yu Zhanqing, et al. Electric field step in air gap streamer discharge [J]. Applied Physics Letters. 2011, 99: 221503. 